\title{
Evidence of a pharmacodynamic EEG profile in rats following clonidine administration using a nonlinear analysis
}

\author{
David-Olivier D Azulay ${ }^{1 *}$, Benjamin Renoux ${ }^{2}$ and Magnus Ivarsson ${ }^{1}$
}

\begin{abstract}
Background: Changes caused by clonidine in rodent electroencephalograms (EEG) have been reported with some inconsistency. For this reason, a pre-clinical study was conducted in order to confirm previous findings with both a standard spectral analysis and a sleep stage scoring procedure. In addition, a nonlinear technique for analysing the time-varying signals was implemented to compare its performance against conventional approaches.
\end{abstract}

Results: The nonlinear method succeeds in quantifying all dose-related responses from the data set relying solely on the EEG trace.

Conclusions: Nonlinear approaches can deliver a suitable alternative to the sleep-stage scoring methods commonly used for drug effect detection.

\section{Background}

The role of the noradrenergic system in sleep physiology has been studied extensively using different pharmacological approaches [1-5]. It is well established that the non-selective alpha- 2 agonist clonidine promotes nonrapid eye movement (NREM) sleep in both humans and rats mainly by activating presynaptic inhibitory autoreceptors and thereby inhibiting noradrenergic neurotransmission. However the specific changes caused by clonidine directly in the EEG is less clear both in humans and rats.

In humans, clonidine has been shown to cause changes in the EEG [6-8]. have noticed that power is increased in the $1.0-4.0 \mathrm{~Hz}$ range while decreased in the 8.0-12 Hz range. Recently published data suggest that clonidine causes different effects on the EEG depending on level of exposure [9]. In this study the authors showed a significant decrease in power in the $0.5-12 \mathrm{~Hz}$ band during rapid eye movement (REM) sleep only.

The findings with clonidine from rodent EEG studies are equally ambiguous [10]. showed that clonidine caused the power to decrease in the $0.1-4 \mathrm{~Hz}$ range and

\footnotetext{
*Correspondence: david-olivier.azulay@wanadoo.fr

'Pfizer Global Research and Development, Ramsgate Road, Sandwich, CT13 9NJ, UK

Full list of author information is available at the end of the article
}

increase in the 4.1-8 $\mathrm{Hz}$ range compared to vehicle controls specifically in the NREM sleep stage. In contrast, [11] showed that the changes caused by clonidine were characterized by a significant increase in nearly all the frequency range from $1-30 \mathrm{~Hz}$, with a peak at $13 \mathrm{~Hz}$. Whether these differences in the reported pre-clinical and clinical findings with clonidine are only due to varying exposure levels or whether there are other additional causes (e.g. definitions of bands in the human and rodent studies, varying electrode derivations, changes restricted to specific sleep stages) is unclear.

An EEG recording is the superposition of a number of electrical signals eminating from various regions in the brain. If all these activities generated perfect superpositions of electrical sine wave oscillations then a linear method like a Fourier transform would expose these periodic components [12]. In the case of non-regular time-varying signals, the task is considerably more complex and requires several steps. Nonlinear approaches aim first at detecting if a deterministic structure exists in the waveform before any further calculations. The presence or absence of determinism in a given data set is the corner stone of its numerical analysis since it completely defines which category of algorithms is practicable. 
In terms of system dynamics, time series EEGs can be viewed as 2-dimensional windows open to a $m$-dimensional state space where time points repeat complex cyclic patterns [13]. Since a reliable approximation of $m$ would help understand the underlying mechanisms involved, algorithms designed to estimate this dimension have been applied [14-16]. Although none of the tested algorithms was able to deliver a definitive answer so far, these numerical methods are capable of extracting useful parameters for very specific phases like sleep [17-19], epileptic [20], schizophrenic [21] or anaesthesia [22] stages. These examples suggest that the measurement of the EEG determistic aspect can quantify different episodes of brain activities by using nonlinear methods.

The aim of this study was to identify a sensitive nonlinear model of EEG analysis that can extract the pharmacodynamic signal of clonidine from EEG recordings from freely moving animals.

\section{Materials and methods}

All animal experiments were carried out in accordance with the United Kingdom Animals (Scientific Procedures) Act 1986 and associated guidelines and approved by the local ethics committee. Rats were implanted with radiotelemetry transmitters (Data Sciences International, St Paul, MN, USA) intraperitoneally under isoflurane anaesthesia for the recording of the EEG and electromyogram (EMG). The cortical EEG electrodes (stainless steel screw electrodes) were implanted epidurally over the left parietal cortex $(2.0 \mathrm{~mm}$ anterior and $2.0 \mathrm{~mm}$ lateral to lambda) and over the left frontal cortex $(2.0 \mathrm{~mm}$ anterior and $2.0 \mathrm{~mm}$ lateral to bregma) for a fronto-parietal EEG recording [23-25]. The electrodes and leads attached to the skull were covered with dental acrylic and a second pair of electrodes was attached to the neck muscles to measure general EMG activity needed for the sleep stage analysis only. The rats were allowed to recover from the implantation of the device for at least 2 weeks, and the experiment was started once the animals were certified fit to continue by a veterinary surgeon. Animals were singly housed on a standard 12$12 \mathrm{~h}$ light-dark cycle and received standard diet and water ad libitum.

EEG and EMG data were continuously sampled at 250 $\mathrm{Hz}$ and the spectral upper limit was set at $40 \mathrm{~Hz}$, with Data Sciences International hardware and software for 12 hours immediately following administration of drug at light onset. Animals were orally dosed with 0.03 (low dose (LD)), 0.1 (medium dose (MD)) or 0.3 (high dose (HD) $\mathrm{mg} / \mathrm{kg}$ clonidine or vehicle (VC) $(0.5 \% \mathrm{w} / \mathrm{v}$ methylcellulose $+0.1 \% \mathrm{v} / \mathrm{v}$ Tween 80 ) at light on-set in a four way cross-over design with at least 48 hours between each dose. Recording of EEG and EMG signals began immediately after dosing. In addition to the
Table 1 Sleep stage scoring principle

\begin{tabular}{rll}
\hline mod & low EEG & high EEG \\
\hline low EMG & REM & NREM \\
high EMG & WAKE & WAKE \\
\hline
\end{tabular}

The modulus (mod) is defined as the absolute value of the area under the curve from the EEG or EMG traces.

sampling of these two signals, a general activity measure and core body temperature were collected in parallel. Due to an archiving issue, not all the files were available for the analysis.

Besides the nonlinear technique detailed below, a sleep stage analysis was completed to identify potential correlations between both outputs. A sleep stage discriminator was programmed whose logic is similar to a visual analysis [24]: its algorithm is summarised in Table 1.

EEG traces were cut into 16.384 second epochs $\left(2^{12}\right.$ points). Since one epoch of EEG data values tend to follow a gaussian distribution the Kolmogorov-Smirnov test was used to filter artefacts [26]: $2 \%$ of the epochs were rejected from our data set. The integral local deformation (ILD) time-embedding window algorithm was implemented to evaluate the deterministic structure of the data [27]; in this work a validation was carried out against known systems [28].

For a given dimension $m$ and time delay $\tau$, the signal is represented by points $\mathbf{x}(t)=\left[v_{t}, v_{t+\tau}, \ldots v_{t+(m-1) \tau}\right]^{T}$ whose displacements are tracked and quantified according to the homogeneity of their flow. If some unknown but deterministic effects drive the data, they should produce a small perturbation to a moving cloud of points as time passes by: points on neighbouring trajectories remain neighbouring points for small evolution times $\Delta$ as sketched in Figure 1[27]. The ILD algorithm calculates the average deviation in terms of distance between points in a cloud for a given $(m, \tau)$ couple (up to a normalisation factor).

To allow comparisons with published results, the data analysis relied on a $0-40 \mathrm{~Hz}$ power spectrum to identify drug-related signals in the raw EEG data. Efforts were concentrated in distinguishing a clear effect in the EEG down to the lowest administered dose of clonidine.

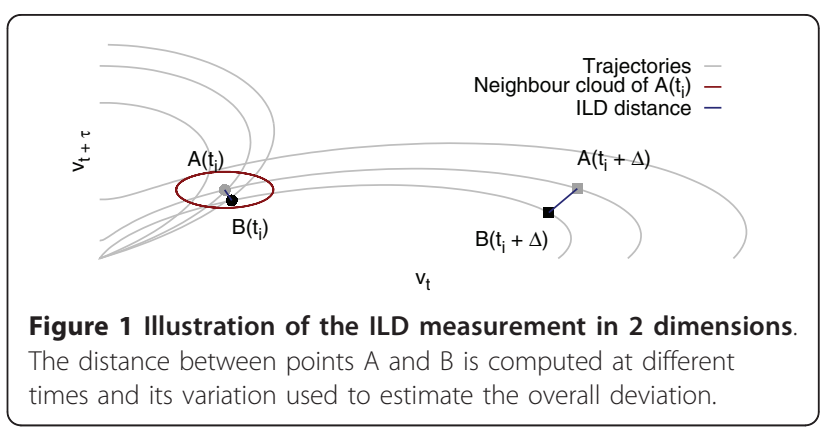



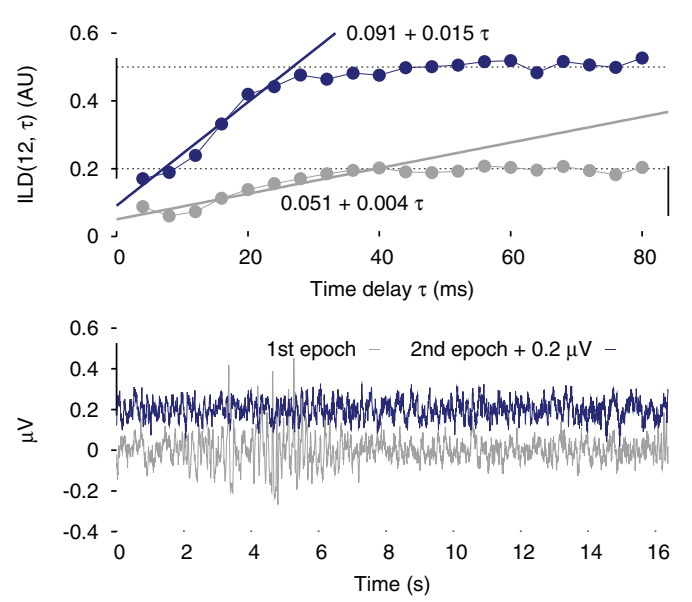

Figure 2 Results of the ILD calculations on two different epochs from rat 1/VC. Since a high plateau is reached faster than a low one, the slope $b$ correlates with the level of the plateau.

\section{Results}

The ILD procedure was run on every EEG epoch for each dimension $m \in[2,12]$ and time delay $\tau \in[4,80]$ in $\mathrm{ms}$ (in $4 \mathrm{~ms}$ increment) as input parameters. Plots identical to Figure 2 were generated to perform visual verification; from these data two groups were identified:

1. where the plateau of the graph is lying high around 0.5 .

2. where the plateau of the graph is lying low around 0.2 .

From a time-embedding perspective there is no local minimum, that is to say no time delay $\tau$ which induces a minimal deviation. The ILD curves systematically reach a plateau after a few iterations. The dimension was arbitrarily fixed at $m=12$ since all the plots produced with a dimension estimation $m$ greater than 10 had their plateaux converging.

Nevertheless these plots provide a quantification of the structure of the signal and details are presented on how the steepness of the slope preceding the plateau is a valuable measurement in terms of characterisation of the time series. The ascending part can be modelled with a simple linear $a+b \tau$ fit of the first 6 points. The value of $b$ is therefore a measure of both the rate of the convergence of the ILD computations and the height of the plateau. If this nonlinear derived parameter $b$ is overlapped with the activity channel to investigate correlations, as shown in Figure 3, four distinct (activity, $b$ ) combinations are possible:

1. (null activity, high $b$ ) periods are regular throughout the experiment (e.g. time intervals B, $\mathrm{M}, \mathrm{O}, \mathrm{S}$ ). They explain most of the main wide peaks in the plot.

2. (null activity, low $b$ ) periods are distributed over the time course (e.g. time intervals A, D, F, H, J, L, $\mathrm{N}, \mathrm{P}, \mathrm{R}, \mathrm{T}, \mathrm{V})$. They cause the recurrent flat parts in the plot.

3. (non-null activity, high $b$ ) periods are quite short and randomly localized (e.g. time intervals C, E, G, I, $\mathrm{K}, \mathrm{Q}, \mathrm{U})$, generating rather sharp peaks.

4. (non-null activity, low $b$ ) periods are quasi inexistent in the experiments.

To demonstrate the potential of $b$ at this early part of the analysis, a 30-minute window (from 10 to $40 \mathrm{~min}$ utes) displays in all rats an average value that already produces an inverse dose-response relationship (see Figure 4). This time frame is consistent with the pharmacokinetic properties of clonidine reported by $[29,30]$ : clonidine dosed at $0.25 \mathrm{mg} / \mathrm{kg}$ peaks in the brain within 2 min and disappears at an average half-life rate of 70 $\min$.

The discriminant function of $b$ is improved by pairing it with the normalised power of the $\delta$ band, defined as the ratio of the power of the $0.5-4 \mathrm{~Hz}$ band to the $0-40$ $\mathrm{Hz}$ band from a Fourier transform based spectrum. Using $\delta$ as the abscissa and $b$ as the ordinate for each epoch, vehicle plots display two clusters (WAKE/REM vs NREM) whilst drug plots display mainly a diffused

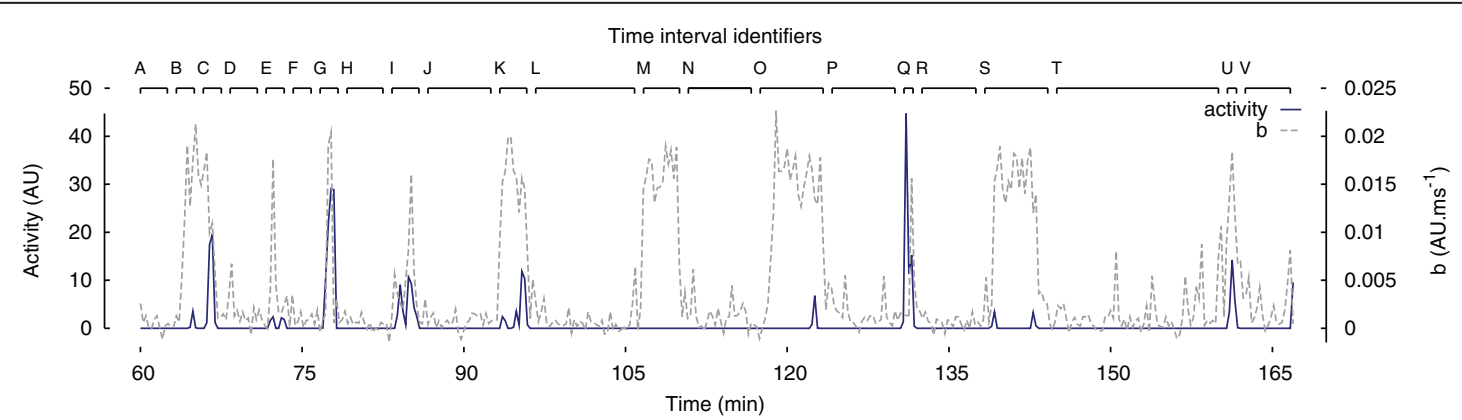

Figure 3 Activity and $b$ overlap for rat $1 /$ VC. Some recurring patterns are visible throughout the time course. 


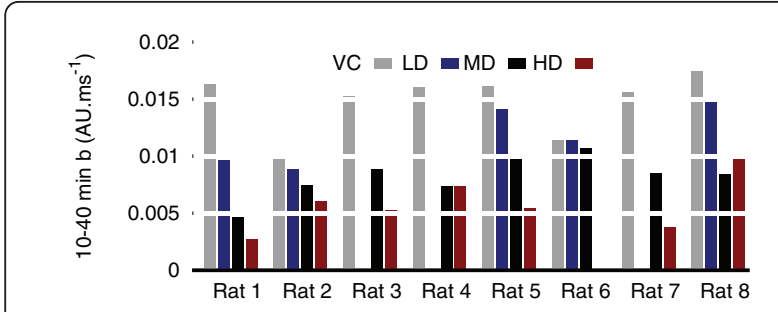

Figure 4 Inverse dose-response signal obtained with the slope parameter $b$ within the 10-40 minute window.

cluster during the first six hours of the experiment as seen Figure 5.

These two-dimensional patterns were quantified using a fuzzy $k$-means clustering algorithm [31] taking into account the following assumption: a 2 cluster input data set, expected for the baseline condition, is bound to produce an outcome very different from a 1 cluster input data set, expected for a drug effect, if fed into a 2 cluster search. Each point is given a belonging probability proportional to the inverse of its distance from the center of a cluster. A clear-cut cluster gathers points with high probabilities, that is to say close to its center, whereas a diffused one encompasses points further away. Hence, the quality of the output clustering is the measure of interest: the area covered by the two clusters becomes smaller when the amount of drug increases as seen in Figure 6, but the total number of points remains approximately the same. The density of the clusters is then greater with a drug onboard. This is the reason why the average cluster density $(\mathrm{ACD})$ criterium renders the clear dose-response relationship observed in Figure 7.

Finally, the level of confidence in the $(\delta, b)$ couple is raised to a higher degree if the response exhibits a pharmacodynamic time response. A 6 hour sliding time window reveals that the proposed measurement is quite suitable as a pharmacological biomarker: Figure 7 shows the evolution of the ACD over 12 hours for all 8 rats. Since time is now a proper dimension, the curves can be interpreted as pharmacodynamic profiles: the stacking order of the escalated doses is preserved and the drug effect disappears progressively with time. For the sake of comparison, sleep stage distributions shown in Figure 8 are more difficult to interpret.

\section{Discussion}

A common technique to detect drug impacts on EEGs relies on sleep stage scores [32]: time proportions of sleep stage periods [24] or powers per bands [23,32] have demonstrated pharmacological effects. Ideally, from a cost and time perspective, the pharmacodynamic effect of a novel drug should be assessed in parallel to standard first in human (FIH) trials. This could potentially be done with a sensitive automatic analysis based on standard wake EEG recordings. However clinical sleep architecture studies tend to be more expensive, mainly due to the time it takes to run these studies in specialist sleep centres. The detection of a pharmacological response directly from the EEG trace would therefore be the preferred solution in an early clinical trial, if achievable.

Nevertheless, links to sleep stages are detailed here for comparison purposes but not retained as part of the final nonlinear analysis workflow. The normalised powers of the different bands per sleep-wake stage during the control condition also confirm the classic distribution of the different frequency bands. For the vehicle dose, a high power in the $\delta$ band is associated with the

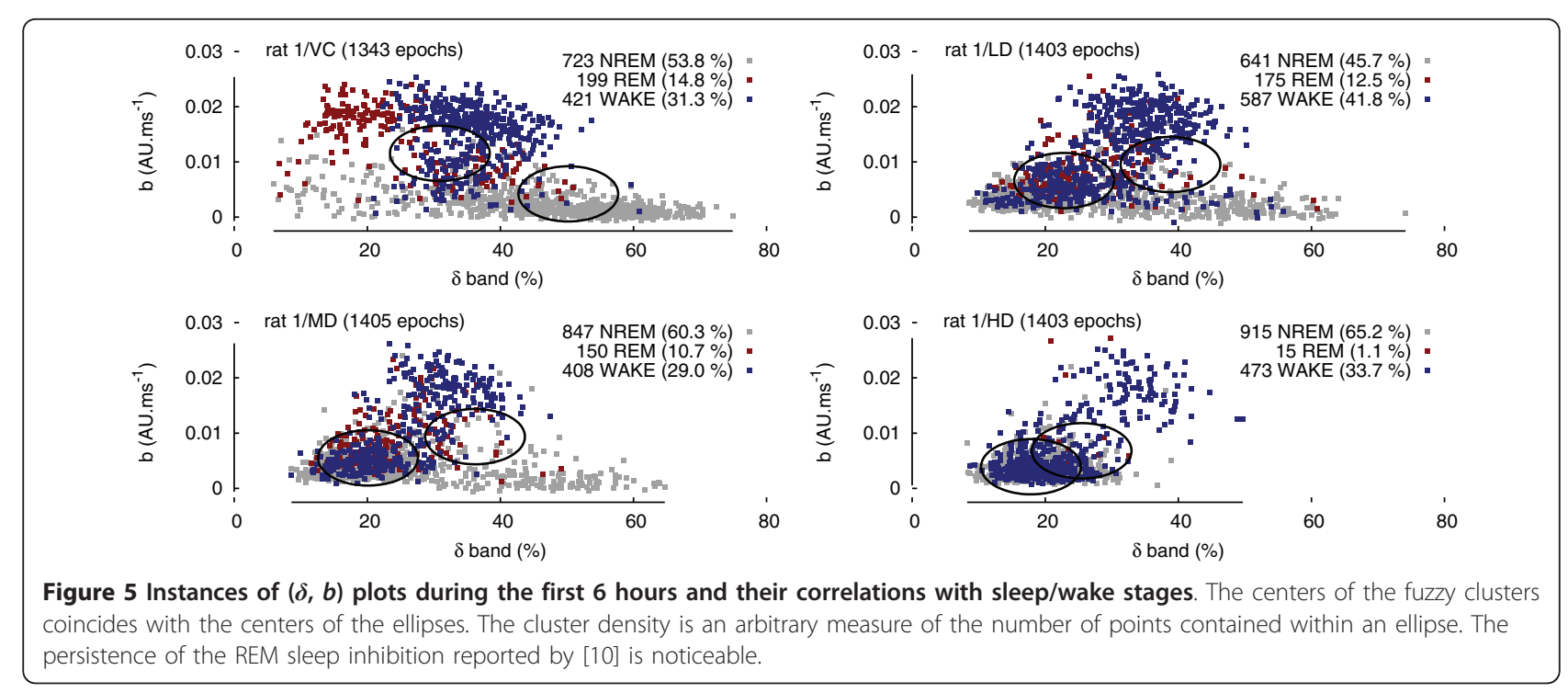




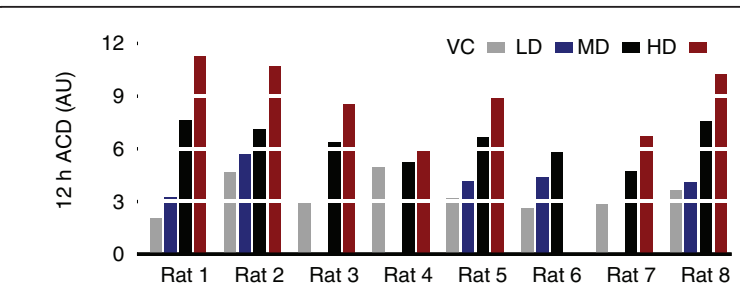

Figure 6 Average of the ACD for the first 12 hours. A clear dose-response signal is visible. The individual cluster densities over time are all plotted in Figure 7.

occurrence of NREM sleep stages and a $\theta$ band power dominates in REM sleep from a fronto-parietal electrode configuration in rodents. The vehicle plot in Figure 5 shows that NREM epochs are synchronised with a high $\delta$ and low $b$ values (bottom right) whereas REM epochs display lower $\delta$ but higher $b$ values (top left). One could potentially rely of the localisation of each point in the different regions of the graph to perform an a posteriori sleep stage epoch scoring.
Clonidine has been shown to reduce the firing of noradrenergic neurons in the Locus Coeruleus (LC) via autoreceptors, thus altering the input to the cortex and causing a change in the texture of the recorded EEG signal, which might be reflected in the non-linear parameter $b[10,33]$.

Table 2 supports the findings described by [10] that the administration of clonidine caused an increase in NREM sleep and a dose dependent decrease in $\delta$ power specifically in NREM sleep rather than a significant increase of the total power as described by [11]. One possible explanation for the discrepancy between the different studies is that [11] recorded the effect of clonidine locally in the prefrontal cortex, while both the outcomes described in this study and those by [10] were carried out with a similar fronto-parietal/occipital electrode configuration.

Given a new drug, there is always a possibility that its unknown effect might corrupt the usual sleep stage classifiers that are well defined for healthy subjects, but less so for preclinical sleep scoring; as an example of
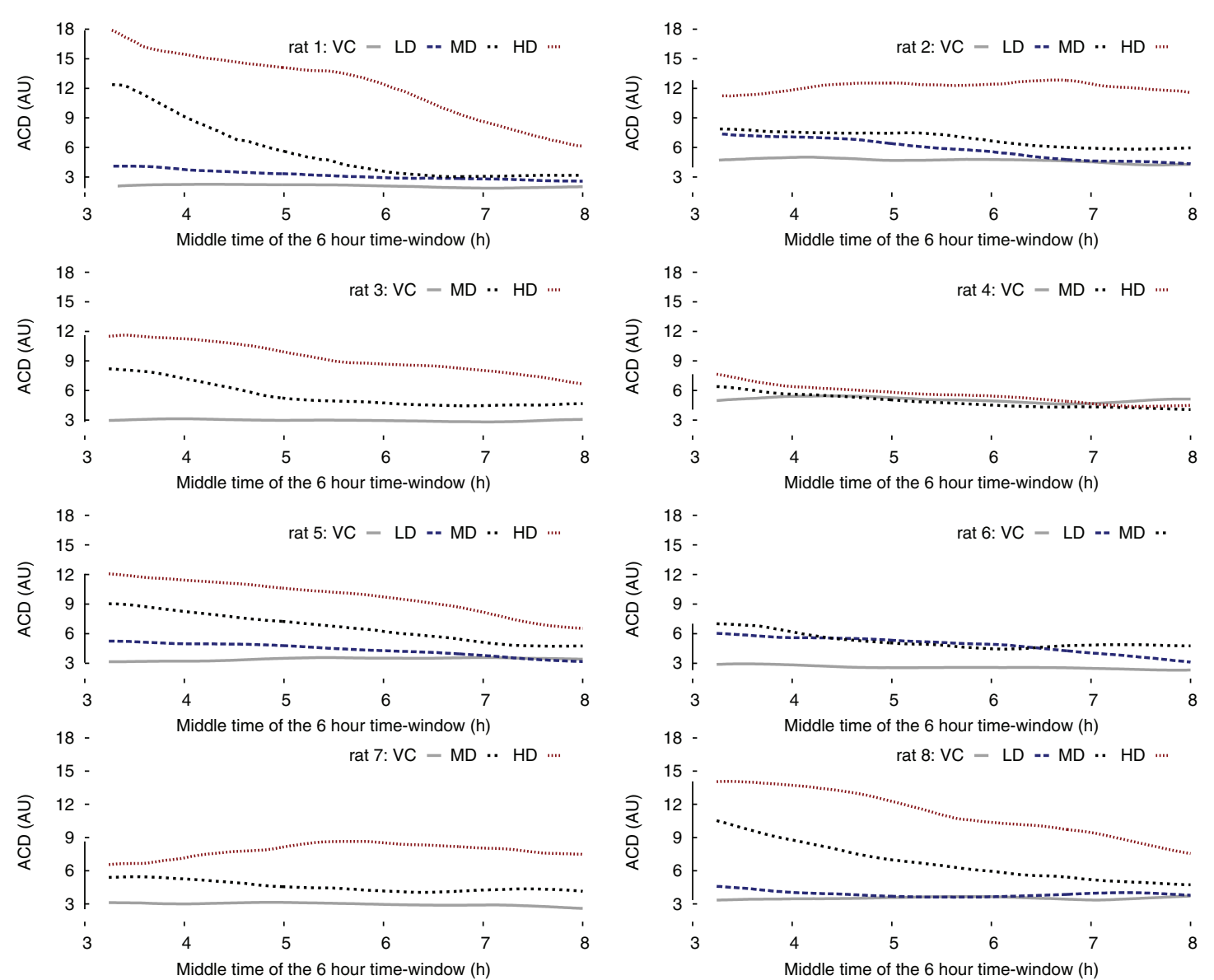

Figure 7 Individual pharmacodynamic profiles obtained from the ACD time courses. The order of the escalating doses is maintained during the different experiments. 

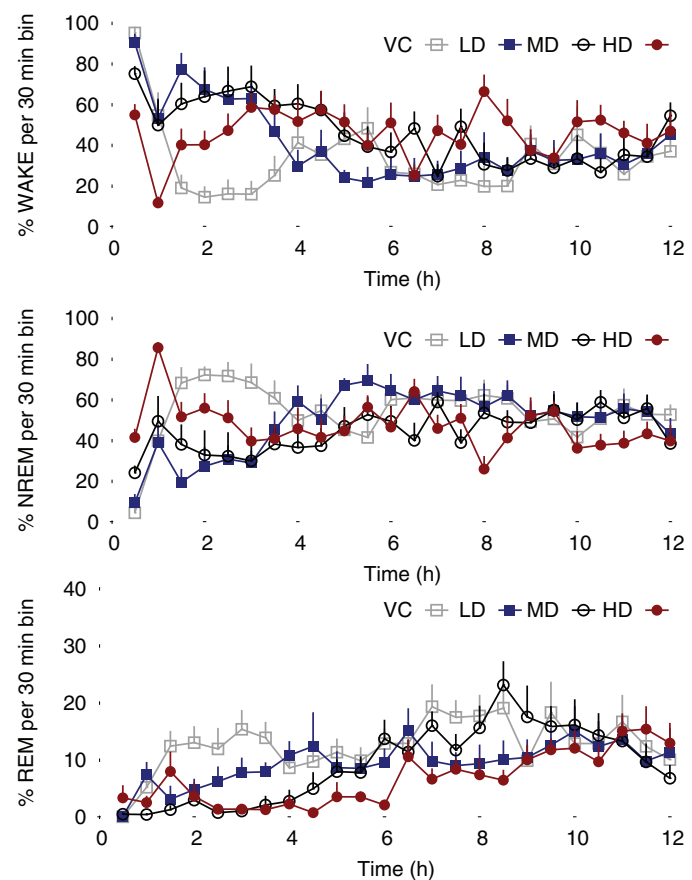

Figure 8 Sleep stage distributions. The NREM "rebound decrease" reported by [10] is visible during the first hour. Nevertheless a significant difference is only observed for the highest dose (see Table 2).

changes in the EEG signal irrespective of sleep stage see [34].

Assumptions solely formed on sleep scores would then be inconclusive. Continuous characterisations like the nonlinear one presented above are likely to be more robust in these particular cases.

This work fully supports the idea that nonlinear techniques are valuable solutions for analysing EEG. The results presented here are consistent as clonidine is known to affect sleep [35] and nonlinear approaches are known to discern sleep stages [28]. The combination with the standard $\delta$ band commonly associated with the awake stage reinforces the plausibility of having extracted a genuine signal from this data set.
In conclusion, this study describes a generic mathematical framework that can extract a pharmacodynamic profile of clonidine from raw EEG data collected from a fronto-parietal electrode derivation in freely-moving rodents, which could offer an alternative approach to study drug effects in early clinical trials. The model is based on a numerical analysis tool whose field of application is nonlinear dynamic systems. This innovative approach can potentially provide a translatable analysis methodology for assessing central pharmacodynamic effect and bridge the preclinical and clinical EEG observations.

\section{Author details}

${ }^{1}$ Pfizer Global Research and Development, Ramsgate Road, Sandwich, CT13 9NJ, UK. Ecole des Mines d'Alès, Avenue Clavières, 30319, Alès, France.

\section{Authors' contributions}

DOA and BR have developed and implemented the algorithms used in the analysis. $\mathrm{MI}$ conducted the rat experiments. DOA and MI helped to draft the manuscript. All authors read and approved the final manuscript.

\section{Competing interests}

$\mathrm{DOA}$ and $\mathrm{Ml}$ receive salaries from Pfizer.

Received: 11 March 2011 Accepted: 26 June 2011

Published: 26 June 2011

\section{References}

1. Aston-Jones G, Chiang C, Alexinsky T: Discharge of noradrenergic locus coeruleus neurons in behaving rats and monkeys suggests a role in vigilance. Progress in brain research 1991, 88:501-520.

2. Aston-Jones $G$, Shipley M, Chouvet $G$, Ennis M, van Bockstaele $E$, Pieribone V, Shiekhattar R, Akaoka H, Drolet G, Astier B: Afferent regulation of locus coeruleus neurons: anatomy, physiology and pharmacology. Progress in brain research 1991, 88:47-75.

3. Berridge CW: Noradrenergic modulation of arousal. Brain Research Reviews 2008, 8:1-17.

4. De Sarro G, Ascioti C, Froio F, Libri V, Nisticò G: Evidence that locus coeruleus is the site where clonidine and drugs acting at alpha 1- and alpha 2-adrenoceptors affect sleep and arousal mechanisms. British Journal of Pharmacology 1987, 90:675-685.

5. Nisticó G, De Sarro G, G GB, Mollace V: Altered sensitivity of alpha 2 adrenoceptors in the brain during aging in rats. Annals of the New York Academy of Sciences 1992, 673:206-213.

6. Itil TM, Itil KZ: Central mechanism of clonidine and propranolol in man. Chest 1983, 83:411-416, [Supplement].

7. Bischoff P, Scharein E, Schmidt GN, von Knobelsdorff G, Bromm B, am Esch JS: Topography of clonidine-induced electroencephalographic

Table 2 Averaged normalised powers in spectral bands for the distinct doses and sleep-wake stages during the first hour

\begin{tabular}{|c|c|c|c|c|c|c|c|c|c|c|c|c|c|c|c|}
\hline \multicolumn{6}{|c|}{ NREM powers in $\%$} & \multicolumn{5}{|c|}{ REM powers in \% } & \multicolumn{4}{|c|}{ WAKE powers in $\%$} & \multirow[b]{2}{*}{$\% / h$} \\
\hline & $\delta$ & $\theta$ & $\alpha$ & $\beta$ & $\% / h$ & $\delta$ & $\theta$ & $\alpha$ & $\beta$ & $\% / h$ & $\delta$ & $\theta$ & $\alpha$ & $\beta$ & \\
\hline VC & 33.2 & 26.3 & 16.2 & 14.4 & 28.5 & 20.4 & 37.8 & 13.8 & 11.7 & 4.3 & 31.2 & 28.3 & 11.8 & 11.5 & 67.2 \\
\hline LD & 30.2 & 28.7 & 16.4 & 14.9 & 27.5 & 25.9 & 30.8 & 13.0 & 14.1 & 3.5 & 28.3 & 30.7 & 12.2 & 12.4 & 69.0 \\
\hline MD & 29.6 & 30.3 & 15.8 & 15.0 & 39.1 & 26.8 & 29.1 & 13.3 & 15.2 & 0.7 & 27.1 & 32.1 & 12.6 & 13.4 & 60.2 \\
\hline$H D$ & 27.6 & 33.6 & 15.8 & 14.7 & $65.7^{\dagger}$ & 25.7 & 31.5 & 13.6 & 15.1 & 4.1 & 26.3 & 33.6 & 13.6 & 14.2 & $30.2^{\dagger}$ \\
\hline
\end{tabular}

Powers in bold indicate the maximum of the four band powers $(\delta=0.5-4 \mathrm{~Hz}, \theta=4-8 \mathrm{~Hz}, \alpha=8-12 \mathrm{~Hz}$ and $\beta=12-20 \mathrm{~Hz}$ ) for each stage and dose. The percentage of time spent during the first hour $(\% / \mathrm{h})$ per stage and dose is reported as well. Significant difference $(p<0.05)$ between VC and HD (comparison made using a one-way ANOVA) is marked with the + symbol. 
changes evaluated by principal component analysis. Anesthesiology 2000, 72:1545-1552

8. Bischoff P, Schmidt GN, Scharein E, Bromm B, am Esch JS: Clonidine induced sedation and analgesia. Journal of Neurology 2004, 251:219-221.

9. Miyazaki S, Uchida S, Mukai J, Nishihara K: Clonidine effects on all-night human sleep: Opposite action of low- and medium-dose clonidine on human NREM-REM sleep proportion. Psychiatry and Clinical Neurosciences 2004, 58:138-144.

10. Seidel WF, Maze M, Dement WC, Edgar DM: Alpha-2 adrenergic modulation of sleep: time-of-day-dependent pharmacodynamic profiles of dexmedetomidine and clonidine in the rat. Journal of Pharmacology and Experimental Therapeutics 1995, 275:263-273.

11. Sebban C, Zhang XQ, Tesolin-Decros B, Millan M, Spedding M: Changes in EEG spectral power in the prefrontal cortex of conscious rats elicited by drugs interacting with dopaminergic and noradrenergic transmission. British Journal of Pharmacology 1999, 128:1045-1054.

12. Klonowski W: Everything you wanted to ask about EEG but were afraid to get the right answer. Nonlinear Biomedical Physics 2009, 3:2

13. Stam C: Nonlinear dynamical analysis of EEG and MEG: review of an emerging field. Clinical Neurophysiology 2005, 116:2266-2301.

14. Grassberger P, Procaccia I: Measuring the strangeness of strange attractors. Physica 1983, 189-208.

15. Rapp P, Zimmerman I, Albano A, Deguzman G, Greenbaun N: Dynamics of spontaneous neural activity in the simian motor cortex: The dimension of chaotic neurons. Physics Letters A 1985, 110(6):335-338.

16. Mayer-Kress G, Layne SP: Dimension of the Human Electroencephalogram. Annals of the New York Academy of Sciences 1987, 504:62-87.

17. Pereda E, Gamundi A, Rial R, González J: Non-linear behaviour of human EEG: fractal exponent versus correlation dimension in awake and sleep stages. Neuroscience Letters 1998, 250:91-94.

18. Kobayashi T, Misaki K, Nakagawa H, Madokoro S, Ota T, Ihara H, Tsuda K, Umezawa Y, Murayama J, Isaki K: Correlation dimension of the human sleep electroencephalogram. Psychiatry and Clinical Neuroscience 2000, 54:11-16.

19. Shen Y, Olbrich E, Achermann P, Meier P: Dimensional complexity and spectral properties of the human sleep EEG. Clinical Neurophysiology 2003, 114:199-209.

20. Kunhimangalam R, Joseph P, Sujith O: Nonlinear analysis of EEG signals: Surrogate data analysis. Recherche et Ingénierie Biomédicale 2008, 29:239-244.

21. Lee YJ, Zhu YS, Xu YH, Shen MF, Zhang HX, Thakor N: Detection of nonlinearity in the EEG of schizophrenic patient. Clinical Neurophysiology 2001, 112:1288-1294

22. Widman G, Schreiber T, Rehberg B, Hoeft A, Elger C: Quantification of depth of anesthesia by nonlinear time series analysis of brain electrical activity. Physical Review E 2000, 62:4898-4903.

23. Kantor $S$, Jakus R, R RB, Halasz P, Bagdy G: Acute and long-term effects of the 5-HT2 receptor antagonist ritanserin on EEG power spectra, motor activity, and sleep: changes at the light-dark phase shift. Brain research 2002, 943:105-111.

24. Ivarsson M, Paterson LM, Hutson PH: Antidepressants and REM in WistarKyoto and Sprague-Dawley rats. European Journal of Pharmacology 2005, 522:63-71.

25. Paterson LM, Wilson SJ, Nutt DJ, Hutson PH, Ivarsson M: A translational, caffeine-induced model of onset insomnia in rats and healthy volunteers. Psychopharmacology 2007, 191:943-950.

26. Delorme A, Makeig S: EEGLAB: an open source toolbox for analysis of single-trial EEG dynamics including independent component analysis. Journal of Neuroscience Methods 2004, 134:9-21[Http://sccn.ucsd.edu/eeglab/ ].

27. Buzug T, Pfister G: Optimal delay time and embedding dimension for delay-time coordinates by analysis of the global static and local dynamical behavior of strange attractors. Physical review A 1992, 45(10):7073-7088.

28. Galka A: Topics in nonlinear time series analysis with implications for EEG analysis Advanced series in nonlinear dynamics, World Scientific; 2000

29. Conway EL, Jarrott B: Clonidine distribution in the rat: temporal relationship between tissue levels and blood pressure response. British Journal of Pharmacology 1980, 71:473-478.
30. Conway EL, Jarrott B: Tissue Pharmacokinetics and Biopharmaceutics. Journal of Pharmacokinetics and Biopharmaceutics 1982, 10(2):187-200.

31. Duda RO, Hart PE, Stork DG: Pattern classification. second edition. John Wiley and Sons; 2001.

32. Bjorvtan B, Fagerland S, Ursin R: EEG power densities $(0.5-20 \mathrm{~Hz})$ in different sleep-wake stages in rats. Physiology and Behavior 1998, 63:413-417.

33. Mallick BN, Singh S, Pal D: Role of alpha and beta adrenoceptors in locus coeruleus stimulation-induced reduction in rapid eye movement sleep in freely moving rats. Behavioural Brain Research 2005, 158:9-21.

34. Winsky-Sommerer R, Vyazovskiy W, Homanics GE, Tobler I: The EEG effects of THIP (Gaboxadol) on sleep and waking are mediated by the GABA $\delta$-subunit-containing receptors. European Journal of Neuroscience 2007, 25:1893-1899.

35. Prince JB, Wilens TE, Biederman J, Spencer T, Wozniak JR: Clonidine for sleep disturbances associated with attention-deficit hyperactivity disorder: a systematic chart review of 62 cases. Journal of the American Academy of Child and Adolescent Psychiatry 1996, 35:599-605.

doi:10.1186/1753-4631-5-4

Cite this article as: Azulay et al.: Evidence of a pharmacodynamic EEG profile in rats following clonidine administration using a nonlinear analysis. Nonlinear Biomedical Physics 2011 5:4.

\section{Submit your next manuscript to BioMed Central and take full advantage of:}

- Convenient online submission

- Thorough peer review

- No space constraints or color figure charges

- Immediate publication on acceptance

- Inclusion in PubMed, CAS, Scopus and Google Scholar

- Research which is freely available for redistribution

Submit your manuscript at www.biomedcentral.com/submit
Ciomed Central 